\title{
PROYECTO DIOCESANO DE PASTORAL VOCACIONAL
}

DOI: https://doi.org/10.52039/seminarios.v52i180.666

Autor: Archidiócesis de MéridaBadajoz.

1. Origen y razón de ser del Proyecto.

Durante el curso 2001-2002, el Equipo de Formadores del Seminario, recogiendo la sensibilidad y la reflexión de los sacerdotes que en los últimos años han ejercido esa misma responsabilidad, elaboró una reflexión sobre la realidad actual del Seminario y de la acción pastoral diocesana encaminada a suscitar vocaciones al presbiterado.

Según esta reflexión dos datos aparecen de forma evidente:

- el numero de chicos en el Seminario Menor desciende notablemente.

- el numero de seminaristas menores que acceden al Mayor es escaso en sí mismo y en relación con los seminaristas mayores que llegan desde otros ámbitos o procesos.

Esto hizo que el Consejo Episcopal y el Equipo del Seminario consideraran necesario trasladar el tema al Consejo del Presbiterio con la inten- 
ción de compartir la inquietud y enriquecer la reflexión. El 17 de octubre de 2002 el Presbiterio acoge el asunto y los consejeros se pronuncian sobre él.

Durante el curso 2002-2003 en todos los arciprestazgos se dedica al menos una sesión de trabajo al mismo documento, con la pretensión de que el Presbiterio Diocesano se pronunciara sobre las causas de la falta de vocaciones en la actual situación de nuestra Archidiócesis, sugiriera las líneas de actuación que habría que plantearse para motivar y fomentar la pastoral vocacional al ministerio presbiteral y aportara medios concretos para impulsar la acción pastoral orientada a la promoción vocacional al presbiterado en los niveles diocesano, arciprestal y parroquial.

Como fruto de todo lo anterior se pudo observar una importante sensibilización en el conjunto de los sacerdotes sobre la pastoral vocacional en orden al ministerio presbiteral. Del mismo modo este proceso dio lugar a sugerencias concretas de acción que no pueden quedarse aparcadas, ni pueden ponerse en marcha como acciones puntuales sin una articulación clara.

El Plan Diocesano de Animación Vocacional, que se pone en marcha desde el Seminario, surge como cauce concreto desde el que sea posible articular los nuevos caminos y los medios de siempre que, para esta tarea, ha sugerido y aportado el conjunto del Presbiterio diocesano.

\section{Principios orientadores del Proyecto}

Este Proyecto tiene de fondo cuatro principios orientadores, que están en su base más profunda y que no pueden olvidarse, en cada una de las acciones concretas que se planteen: confianza inquebrantable, esperanza activa y prioridad pastoral.

a. Llamada desde el Bautismo: la santidad tiene su fundamento en el Bautismo y es para todos los bautizados en Cristo. El ser humano, en efecto, es «llamado» a la vida y al venir a la vida, lleva y encuentra en sí la imagen de Aquél que le ha llamado. La fidelidad al Bautismo significa, por tanto, mirar a lo alto, como hijos, para llevar a cabo el discernimiento de su voluntad sobre la propia vida y el propio futuro. 
b. Confianza inquebrantable: Las vocaciones presbiterales del futuro serán el fruto de una confianza eclesial que se apoya en la promesa de Dios, como su fundamento. Nuestra Iglesia confía, del mismo modo, en las mediaciones en las que el Señor actúa y que van surgiendo como respuesta a los nuevos tiempos y a las nuevas necesidades. La plegaria por las vocaciones sacerdotales y por este proyecto es una manera inequívoca de expresar que no somos nosotros, sino Dios, la fuente de las vocaciones.

c. Esperanza activa: La iniciativa de Dios no excluye, sino postula, la intervención de la comunidad eclesial. Ella es la mediación fundamental de Dios para toda vocación presbiteral por ello "el deber de fomentar las vocaciones afecta a toda la comunidad cristiana" y no puede, por tanto, concebirse exclusivamente como una actividad específica de algunos organismos o presbíteros "encargados"!. Del mismo modo la pastoral vocacional no es separable de la pastoral general de la Iglesia, sino una dimensión ineludible y central de toda actividad pastoral.

d. Prioridad pastoral: La promoción de vocaciones al presbiterado diocesano, entendida como "suscitar, acoger, acompañar y formar", es, para nuestra Archidiócesis, una prioridad que no admite demora ni paliativos.

\section{Objetivos de Proyecto}

\section{Objetivo General:}

Animar y activar a la Iglesia Diocesana en la promoción de las vocaciones, especialmente en la referida al ministerio ordenado y a la vida consagrada.

1. Cf. Pdv. 41.

Primer Congreso internacional sobre la vocaciones. Documento conclusivo, 1973, n.5. 


\section{Etapas:}

\section{SENSIBILIZACIÓN}

\section{Objetivos específicos:}

1. Sensibilizar a los sacerdotes y agentes de pastoral, para que todos descubran la importancia de introducir en los procesos y acciones pastorales, el planteamiento -la pregunta-, vocacional.

2. Ayudar y orientar en la etapa de sensibilización, a los agentes de pastoral, con medios concretos, para que puedan suscitar en todos/as la cuestión vocacional.

\section{PROFUNDIZACIÓN}

\section{Objetivo Específico:}

Acompañar y profundizar el proceso de maduración de la escucha y la respuesta vocacional.

Progresar en el proceso de maduración vocacional en quienes se descubran indicios de llamada al ministerio ordenado o a la vida consagrada.

Acciones del Proyecto.

\section{ETAPA DE SENSIBILIZACIÓN:}

\section{Destinatarios:}

Sacerdotes.

Profesores de religión (de primaria y secundaria).

Animadores de adolescentes, jóvenes.

Responsables de Movimientos.

Catequistas.

Familias. 


\section{Medios:}

A nivel general:

Convivencias Vocacionales por zonas y campamento vocacional. P. Damasco

Visita al Seminario Metropolitano. P. "Venid y lo Veréis".

"Publicarteles" trimestrales a parroquias y centros educativos. P. Samuel

Exposición vocacional. P. Samuel.

Presencia en la revista "Seminario" e "Iglesia en Camino". Radio y TV. P. Samuel

Creación de unidades didácticas con el tema vocacional. Delegación de enseñanza P. Samuel.

Creación de unidades catequéticas donde aparezca claramente la cuestión vocacional. Delegación de catequesis. P. Samuel.

Creación de la página Web de pastoral de juventud y vocacional. P. Samuel.

Día del monaguillo. P. Venid y lo Veréis

Campaña vocacional. Día del seminario. P. Samuel

(De entre todo lo que aquí se expone habría que tener en cuenta los medios que no se reducen a acciones puntuales, como los más importantes)

A nivel de zona:

- reunión con sacerdotes y agentes de pastoral (catequistas y profesores de religión) para dar a conocer el proyecto.

- creación del grupo de animadores responsables de las convivencias zonales.

En conexión con otros organismos diocesanos, que faciliten el trabajo con los agentes desde sus estructuras, perteneciendo a los equipos permanentes de los secretariados de enseñanza, vocaciones, juventud y catequesis.

Visitas personales.

Convivencias de ingreso en el seminario como internado.

Acompañamiento personal a chicos/as en clave de orientación. 


\section{ETAPA DE PROFUNDIZACIÓN:}

\section{Destinatarios:}

\section{Chicos}

Desde $6^{\circ}$ de primaria hasta los 18 años, diferenciado por niveles:

Continuidad en el PDAV.

Ingreso en el Seminario Menor internado.

Proceso de acompañamiento en los lugares de origen de los chicos desde la fórmula "seminario menor externo".

Pastoral de juventud.

Movimientos eclesiales.

\section{Medios:}

A partir de $2^{\circ}$ de Bachillerato hasta 30 años:

Continuidad en el PDAV

Seminario Mayor.

Posibilidad de participar en la experiencia "Mar Adentro".

Campo de Trabajo "Buen Samaritano".

Ejercicios espirituales.

Movimientos eclesiales

Pastoral de Juventud.

Chicas

Desde $6^{\circ}$ de primaria hasta los 18 años, diferenciado por niveles:

Continuidad en el PDAV

Experiencia acompañada por CONFER e institutos seculares. "Pozo de Jacob".

Pastoral de Juventud

Movimientos eclesiales.

\section{Medios:}

A partir de $2^{\circ}$ de Bachillerato hasta 30 años:

Posibilidad de participar en la experiencia "Mar Adentro".

Campo de Trabajo "Buen Samaritano".

Ejercicios espirituales.

Noviciado 
Pastoral Juvenil

Movimientos eclesiales.

\section{Agentes de este Proyecto.}

Este Proyecto lo llevará a cabo el Equipo Diocesano de Animación Vocacional.

Este Equipo depende directamente del Arzobispo y de la Vicaría General, del mismo modo que el Seminario.

Tiene como responsable-coordinador a un sacerdote directamente nombrado por el Arzobispo para esta tarea: es el Director del PDAV.

Dadas las razones que originaron el proyecto, y ya que su última finalidad es la promoción vocacional al presbiterado, el Director del PDAV, forma parte del equipo de formadores del Seminario Metropolitano. Su tarea formativa la desarrollará con adolescentes y jóvenes que no están en el internado del Seminario. Del mismo modo los Formadores del Seminario forman parte del PDAV.

Este equipo requerirá la colaboración de los seminaristas del Mayor.

El PDAV está en estrecha colaboración con el Secretariado Diocesano de Pastoral Vocacional, la CONFER y los Institutos Seculares; ya que sus funciones son complementarias. Del mismo modo esta vinculado a los secretariados diocesanos de:

- Catequesis.

- Juventud.

- Enseñanza.

El PDAV necesita contar con una estrecha colaboración del Presbiterio diocesano, especialmente con los responsables arciprestales de pastoral vocacional y con todos los agentes de pastoral que trabajan con niños, adolescentes y jóvenes (catequistas, animadores y profesores de religión). 


\section{Experiencia de sensibilización vocacional}

\section{Proyecto Samuel}

"Trabajo con sacerdotes, profesores de Religión y catequistas"

\section{Justificación:}

En este proyecto, el PDAV quiere hacer unas propuestas de trabajo a los colectivos que están en permanente contacto con niños y jóvenes de nuestra archidiócesis. "Samuel", se ofrece para todos los que están en catequesis, o un sector muy importante como son los alumnos de las clases de Religión que se imparten a lo largo y ancho de nuestra geografía diocesana ya que participan alrededor de 21000 alumnos/as. Creemos que estos alumnos/as es un número suficientemente amplio como para que lo incluyamos en este nuevo impulso que queremos dar a la pastoral vocacional.

\section{Objetivo:}

- Ofrecer claves, para que los agentes de pastoral que trabajan con adolescentes y jóvenes, puedan sugerir la posibilidad de vivir experiencias de encuentro con Dios adaptadas a sus niveles y que permitan una sensibilización a la llamada vocacional.

\section{Destinatarios:}

- Sacerdotes.

- Religiosos y religiosas

- Profesores de Religión de Primaria, ESO y Bachillerato.

- Catequistas.

\section{Exigencias:}

Sensibilidad con lo vocacional en toda su amplitud.

Participar en unos encuentros donde se trabaje y profundice en el "Plan Diocesano de Animación Vocacional", y se llegue a conocer en toda su 
amplitud para descubrir hasta dónde puede llegar la implicación de cada uno.

Participación en el proyecto desde la etapa de sensibilización (Convivencias-Campamentos y "Venid y lo Veréis") hasta la de profundización ("Mar Adentro" o "Campo de Trabajo Buen Samaritano").

\section{Qué les ofrece:}

Formación especifica.

Unidades Catequéticas para trabajar en los procesos de las distintas comunidades.

Web donde encontrar materiales y solucionar problemas planteados.

Exposición de carteles vocacionales con ficha de trabajo para diferentes edades y contextos.

Revista con contenido vocacional.

Materiales para trabajar en el aula. Unidades didácticas para los distintos niveles académicos.

Para los profesores/as de primaria: (hablamos únicamente de $6^{\circ}$ de primaria) una unidad didáctica donde se posibilite la sensibilización y un primer acercamiento a la cuestión vocacional. Materiales con dinámicas y juegos con la cuestión vocacional de fondo, para poder trabajar en el aula durante el año.

Para los profesores/as de secundaria: unidades didácticas y la posibilidad, para los seglares, de participar como monitor/a en las convivencias y campamentos que se ofrecen desde el proyecto de sensibilización vocacional.

Para ambos la posibilidad de acercarse al seminario en un día "de puertas abiertas" previamente ha de aparecer en su programación de área, así pues se ha de comunicar en septiembre.

Posibilidad de ampliar todo lo trabajado en el aula fuera de ella.

La colaboración del profesorado de religión en su totalidad es inestimable, pues posibilita el acercamiento a un número alto de chicos/as y la ocasión de hacerles partícipes de esta experiencia. 


\section{Responsable:}

Responsable será el director de PDAV que coordinará la experiencia y la insertará, desde la etapa de sensibilización, en el Plan de Animación Vocacional de la Diócesis.

\section{Colaboradores:}

Secretariado Diocesano de enseñanza.

Secretariado Diocesano de Catequesis

Sacerdotes.

Religiosos y religiosas que ofrecen este servicio a la Iglesia

Profesores de Religión seglares.

\section{Organización y actividades:}

Dividiremos la archidiócesis en tres zonas:

Zona Oeste: Badajoz, Olivenza, Alburquerque, Montijo y Almendralejo.

Zona Sur: Jerez de los Caballeros, Fregenal de la Sierra, Zafra, Fuente de Cantos y Llerena.

Zona Este: Calamonte, Mérida, Villanueva de la Serena, Zalamea de la Serena y Castuera.

\section{Primera Etapa:}

Encuentro por zonas con los profesores de religión haciéndoles partícipes de las inquietudes que mueven todo el plan de animación vocacional diocesana.

Trabajar junto a la delegación diocesana de enseñanza una serie de unidades didácticas donde se trabaje la pastoral vocacional desde los distintos niveles de enseñanza.

Trabajar junto a la delegación diocesana de catequesis una serie de unidades catequéticas donde se profundice en la pastoral vocacional desde los distintos niveles de catequesis.

Organizar unos encuentros en las distintas zonas para profundizar y descubrir todo lo que subyace en este proyecto, y por contagio, motivar respuestas desde la ilusión e intentar algo nuevo. 


\section{Responsable:}

Responsable será el director de PDAV que coordinará la experiencia y la insertará, desde la etapa de sensibilización en el proyecto global de Animación vocacional de la Diócesis.

\section{Colaboradores:}

Dividiremos la archidiócesis en tres zonas por lo tanto serán tres lo equipos de trabajo para las distintas convivencias.

Serán monitores/as preparados/as, un total de doce por cada zona y divididos por niveles. Contaremos también con la colaboración de seminaristas.

Y sugerimos la necesidad de la participación de los sacerdotes de la zona, para dar mayor continuidad y seguimiento al proyecto.

\section{Organización y actividades:}

Teniendo de fondo la cuestión que nos mueve, es decir, el objetivo general:

Nos proponemos los siguientes objetivos específicos por etapas que trabajaremos en las convivencias y el campamento.

Primera etapa: $6^{\circ}$ de primaria, $1^{\circ}$ de la ESO (11-12 Años).

"Interiorizar que la vocación es una llamada para estar con Jesús comunidad". PFSm

Segunda etapa, $2^{\circ}$ y $3^{\circ}$ de la ESO. (13-14 Años)

"Experimentar que la vocación es un servicio y donación a la comunidad". PFSm

Tercera etapa, $4^{\circ}$ de la ESO y Bachillerato. (15-17 años)

"Profundizar en la vocación como realización personal desde las necesidades de la Iglesia y el mundo". PFSm. 
La experiencia de sensibilización que proponemos solo se puede vivir una vez cada etapa. Si se encuentra en el primer año de una etapa $\left(6^{\circ}\right.$ y $2^{\circ}$ de ESO y $4^{\circ}$ de la ESO) y desea continuar, se le acompaña durante un año para que pueda realizar, al encontrarse en otro nivel, la experiencia siguiente.

Los objetivos de campamento serán los mismos que marcamos en las convivencias pero desarrollados mas ampliamente debido al tiempo con el que contamos. Esta actividad, culmen de la experiencia, la desarrollaremos en el albergue de Gredos que nuestra archidiócesis tiene en Ávila.

\section{Primera Etapa:}

Se elaborarán un material, por niveles, para los grupos de catequesis en los que se trabajará una introducción previa a toda la experiencia de sensibilización, que se le ha pedido al secretariado de catequesis.

También se prepara, para introducir la pastoral vocacional en el aula, una unidad didáctica por niveles de educación, ofrecida a los profesores de religión, parte importante en este proyecto. Estas unidades didácticas estarán elaboradas por el secretariado de enseñanza.

\section{Segunda Etapa:}

Pasar a una etapa directa de profundización:

Seminario menor externo y en paralelo un proceso coordinado por CONFER e Institutos Seculares para las chicas (de 13 a 17 años).

Mar adentro (Mayores de 18 años).

Pastoral de Juventud.

Movimientos Eclesiales

\section{Prolongar la etapa}

Continuamos acompañando en la etapa de sensibilización, y con la posibilidad de participar en el futuro en la etapa de profundización. 


\title{
Lugar de la experiencia:
}

Tres zonas en la diócesis:

Zona Oeste: Badajoz, Olivenza, Alburquerque, Montijo y Almendralejo (a realizar las convivencias en el OSCUS).

Zona Sur: Jerez de los Caballeros, Fregenal de la Sierra, Zafra, Fuente de Cantos y Llerena (a realizar las convivencias en la casa de la Iglesia de Jerez de los Caballeros).

Zona Este: Calamonte, Mérida, Villanueva de la Serena, Zalamea de la Serena y Castuera (a realizar las convivencias en Villagonzalo).

\section{Experiencia de profundización vocacional}

\author{
"MAR ADENTRO"
}

\section{Justificación:}

Partimos de la experiencia llevada a cabo en Milán para sensibilizar y hacer consciente a la Iglesia de la necesidad de responder a la llamada de Dios. En ella se presenta la vocación en toda su amplitud y trabaja la generosidad del que escucha. Estos grupos comenzaron hace veinte años y aún hoy siguen trabajando allí. La experiencia se trasladó a otras diócesis entre ellas Málaga. Los frutos obtenidos en ella nos animan a implantar entre nosotros esta realidad.

\section{Definnición:}

Experiencia de encuentro con Dios, personal y comunitariamente, acompañada por la Iglesia diocesana, en clave de discernimiento del proyecto de vida.

Teniendo de fondo la cuestión "Señor, ¿qué quieres de mí?", se plantea la vocación en toda su amplitud para dar una respuesta y entrar en un proceso hacia la vida sacerdotal, de especial consagración o matrimonial.

\section{Para quiénes:}

Chicos y chicas de 18 a 30 años, que intentan descubrir su vocación, 
que sean capaces de plantearse: ¿Qué quiere Dios de mí? ¿Cómo puedo yo servir mejor a los demás? ¿Cuál es mi vocación?

\section{Objetivo general:}

Mar Adentro pretende ofrecer a los jóvenes un instrumento profundo de discernimiento vocacional. Para aquellas personas que están buscando cuál es su camino: el sacerdocio, la vida religiosa o la vida seglar.

\section{Responsable y colaboradores:}

Responsable será el director del PDAV que coordinará la experiencia y la insertará en el plan global de Animación vocacional de la Diócesis.

\section{Colaboradores:}

- Sacerdotes, religiosos, religiosas y laicos que acompañen los procesos de los jóvenes implicados en la experiencia.

- Sacerdotes, religiosos, religiosas y laicos conocedores de los distintos contenidos que se trabajarán en los encuentros a lo largo de todo el año.

\section{Organización y actividades:}

* Un primer encuentro en el que se inicia al joven en la experiencia. Será un sábado completo.

* A partir de ese momento los jóvenes se reunirán una vez al mes durante la tarde de un sábado con el siguiente esquema de trabajo:

\subsection{Acogida}

\subsection{5 oración inicial.}

16.30 Reunión por grupos, donde compartimos el fruto del trabajo personal, analizamos las dificultades encontradas y exponemos las experiencias obtenidas.

17.30 Exposición del tema de trabajo y formación. A continuación trabajo en grupo.

18.15 Reflexión personal. Oración.

19.15 Descanso 
19.45 Coloquio.

20.30 Oración Personal.

* Algún encuentro mensual podrá realizarse durante todo el sábado completo.

\section{Exigencias:}

- Quienes van a participar envían una carta personal al Obispo donde le exponen los motivos por los que desean implicarse en "Mar Adentro" y donde cuentan su proceso de fe. A su vez ellos o ellas reciben una invitación y asumen 3 compromisos:

1. Asistencia a todos los encuentros.

2. Dedicar un tiempo de oración diaria para trabajar lo dado en el encuentro mensual.

3. Si no tienen acompañante espiritual buscarán uno, que puede ser del grupo de colaboradores o uno que ellos presenten, con el que dialogarán todo lo que acontece durante la experiencia. En la primera sesión donde se le explica el proceso de Mar Adentro, se hará hincapié en las características que ha de tener un acompañante. Una vez al mes dialogarán del proceso con su acompañante.

Al principio de la experiencia con los acompañantes elegidos se tendrá una sesión de trabajo para profundizar sobre el acompañamiento en "Mar Adentro". Y al final una revisión del Proyecto.

\section{Programa:}

Los temas a tratar en los distintos encuentros se pueden organizar de la siguiente manera:

El itinerario vocacional.

El acompañamiento.

La vocación en la Biblia.

La oración y la Lectio Divina.

Las distintas vocaciones.

Material de Verano.

Proyecto Personal de Vida.

Madurez humana

Madurez espiritual.

La experiencia terminará con un fin de semana de ejercicios espirituales. 


\section{Lugar de la experiencia:}

El Seminario Metropolitano San Atón.

Si la realidad nos hace ver que otro lugar es el más idóneo para la experiencia se replantearía en otro sitio.

\section{Experiencia de profundización vocacional}

\section{Seminario Menor Externo}

\section{Una propuesta de "Preseminario"2}

\section{Introducción}

El Plan de Formación para los Seminario Menores de la Conferencia Episcopal Española contempla distintas fórmulas educativas como simultáneas o coexistentes con el Seminario Menor para Internos sólo en casos excepcionales como alternativas al mismo articulándolas en torno a dos ejes o cauces que denomina: Centros Análogos y Preseminario ( ${ }^{\circ} 18,19$ y 20). En él se da por sentado qué condiciones locales y conveniencias pastorales pueden aconsejar su creación y puesta en marcha.

El análisis que hemos hecho de nuestro Seminario Menor y la reflexión en torno a la pastoral vocacional que realizamos durante el curso 20022003 nos orientan hacia esta dirección. Ofrecemos ahora un esbozo de proyecto en torno a lo que podría ser la experiencia de "Seminario menor externo" para nuestra Archidiócesis. Lo hacemos siguiendo los pasos de otras diócesis que ya la tienen iniciada y en algunos casos consolidada.

\section{El Seminario Menor para externos}

Entendemos por "Seminario Menor para Externos" el "lugar" en el que niños, adolescentes y jóvenes, que cursan sus correspondientes estudios académicos en sus colegios y viven con sus familias siguen un proyecto

2 Seguimos fundamentalmente el documento "El Seminario menor para externos", Una nueva experiencia de Seminario menor desde el Plan de Formación de la C.E.E., Tarazona 2001. 
formativo-vocacional, a través de actividades periódicas concretas, animadas y coordinadas por un equipo designado por el Sr. Obispo para esta tarea.

Esta institución no excluye el Seminario Menor interno; quiere ser un apoyo y un agente de pastoral vocacional al servicio del Seminario. Concebimos, por tanto, el Seminario Menor para Externos en nuestra Archidiócesis, como una variante complementaria al Seminario Menor para Internos, que no pretende eliminar éste tal como ahora funciona. Uno y otro constituyen juntos, el mismo y único Seminario Menor Diocesano. Así, pues, el Seminario Menor para Externos, "mutatis mutandis", queda sujeto globalmente al Plan de Formación para los Seminarios Menores de la Conferencia Episcopal Española, en el que se describen:

- la naturaleza, fines, características y relaciones del Seminario Menor;

- los criterios, requisitos y etapas del proceso educativo;

- la formación académica: conexiones y especificidades en relación a los planes y programas oficiales;

- el equipo de educadores: componentes y su preparación, vida y funcionamiento, y su relación con la comunidad diocesana.

3. Sus especificidades más relevantes, en relación con el Seminario Menor para Internos:

\subsection{Para quiénes está pensado}

El Seminario Menor para Externos quiere atender a aquellos chicos, con edades comprendidas entre los once y los dieciocho años, que:

+ cursan sus correspondientes estudios académicos en Centros Públicos o Privados,

+ viven ordinariamente con sus familias,

+ poseen ciertas aptitudes ${ }^{3}$ básicas (exactamente las mismas de un chico interno),

${ }^{3}$ EI PFSm considera aptitudes básicas indispensables: condiciones fisicas y psíquicas normales; suficiente capacidad intelectual para cursar estudios medios; elemental educación en la fe; suficiente sensibilidad religiosa y disponibilidad para participar en los actos de culto; sentido de sociabilidad y de apertura a los demás; capacidad y deseo de colaboración y compromiso (C. PFSm 34). 
+ presentan una inquietud vocacional inicial,

+ son presentados y avalados por el párroco, quien va a tener un papel importante en su seguimiento,

$+y$, a nivel de su Parroquia, si hay posibilidad, participan en algún grupo (catequesis, movimiento, etc.), que sintonice con el programa de formación del Seminario Menor.

\subsection{Qué les ofrece}

+ una ayuda para profundizar su compromiso de fe;

+ unos medios para clarificar su vocación cristiana;

+ un planteamiento para descubrir su posible vocación al sacerdocio.

+ Y todo ello apoyándonos en cinco pilares ${ }^{4}$.

El propio niño o el adolescente que se compromete libremente.

La familia, que colabora con el proyecto.

La parroquia en la que siguen participando y profundizando en su fe.

El grupo vocacional entendido como un espacio y un ambiente de convivencia y formación en fines de semana o en períodos de no actividad académica, de ordinario en las instalaciones del Seminario Menor.

El acompañamiento tanto personal como de grupo "in situ", es decir, en sus ámbitos de estudio y/o residencia.

\subsection{El Equipo de Responsables y Colaboradores}

El Obispo nombra a un sacerdote como primer responsable de un equipo, sería de desear que, en el mencionado Equipo, quedaran integradas con distintos grados de implicación y actuación, como es obvio, las siguientes personas:

a) Responsables directos de esta experiencia serán los Formadores del Seminario Menor para Internos, como organizadores y coordinadores natos, al menos inicialmente.

${ }^{4}$ Cf. Comisión Episcopal de Seminarios y Universidades, "Habla, Señor" Valor actual del Seminario Menor, 1998, 5 a. 
b) Colaboradores:

+ El Responsable Diocesano de Pastoral Vocacional.

+ Los párrocos de los candidatos inscritos en la experiencia, con los que se mantendrá una estrecha colaboración, serán los acompañantes cotidianos y más cercanos al chico.

+ Alguno de los seminaristas mayores, en calidad de animador y monitor de las convivencias de fin de semana, u otras actividades que se programen.

+ Personas que puedan colaborar con clases o talleres, destinados a las convivencias de fin de semana (algún sacerdote o religioso/a, catequista, padres de familia, amigos).

+ Profesores de Religión, Catequistas o Consiliarios en cuyo ámbito de actuación han surgido los candidatos.

+ Algún representante seglar de la comunidad diocesana, sensibilizado con el tema vocacional.

+ El apoyo de alguna persona técnica en Psicología.

\subsection{Organización y actividades:}

El medio fundamental de esta experiencia lo constituyen los encuentros que con una periodicidad mensual se realizarán en el Seminario. Estas convivencias comenzarán los viernes por la tarde y concluirán los domingos después de la comida. Posible esquema:

El viernes se dedicará al estudio y preparación de las tareas escolares. Así como a la revisión de los compromisos marcados en la convivencia anterior.

El sábado por la mañana será dedicado a la formación: charlas, dinámicas, grupos... Por la tarde al deporte y a la reflexión, oración y celebración. También a la convivencia con los seminaristas internos.

El domingo, la Eucaristía centra la mañana. La distribución del material para realizar hasta la convivencia siguiente y la revisión del fin de semana completa el resto de la mañana. Después de comer vienen los padres a recoger a los chicos y se tiene un diálogo personal con ellos (hoy está sustituyendo al encuentro mensual en sus casas).

Otros medios podrán ser retiros o celebraciones especiales.

En verano se tendrá una actividad o un cursillo más amplio.

Puede organizarse algo en las vacaciones de Navidad y Semana Santa. 
Los alumnos del Seminario Menor para Internos participarán en las convivencias y en las actividades integrándose en ellas, como pidan los objetivos planteados, de tal manera que todos sean un único grupo ya que todos son seminaristas.

El Formador nombrado para esta tarea, hará una visita mensual a los chicos en sus respectivos pueblos.

Ya se apuntaba antes que los Párrocos de estos chicos tienen un papel importante, tanto en su desarrollo como en su seguimiento. Al iniciar el curso se convocará a una reunión para comentarles los objetivos, contenidos y metodología del programa educativo. También para decirles lo que se espera de ellos de cara a la consecución de los objetivos de la experiencia. Al final de curso se hará una reunión igual para la evaluación.

En la misma dirección (objetivos, contenidos, metodología, colaboración de ellos y evaluación) se tendrá un diálogo con los padres de los chicos, bien juntos o bien en sus respectivos domicilios.

Los chicos que realizan esta experiencia del Seminario Menor para Externos al igual que los del Seminario Menor para Internos, son para todos los efectos alumnos del mismo y único Seminario Menor, tal como lo estipula la Conferencia Episcopal Española. Por tanto recibirán en sus casas toda la información que les competa y serán convocados, tanto ellos como sus familias, a los actos que prepare el Seminario Menor.

\subsection{Temporalización:}

La experiencia del Seminario Menor para Externos consta de un período suficientemente amplio. A lo largo de este período, o al final de cada curso, se le planteará al alumno la posibilidad de que se integre en el Seminario Menor en internado o que continúe viviendo en su domicilio familiar. Terminados los estudios de Bachillerato o de grado Medio se le ofertará que pase al Seminario Mayor, si el equipo de Formadores lo considera oportuno

\subsection{Exigencias para participar en el Seminario Menor para Externos:}

Estar cursando $6^{\circ}$ de Primaria, E.S.O. o Bachillerato.

Haber pasado por uno o más fines de semana durante el curso anterior para conocer la experiencia. 
Haber manifestado alguna inquietud por el sacerdocio o no descartar el planteamiento vocacional hacia el mismo.

Tener el visto bueno del párroco que es quien presenta al futuro seminarista.

Comprometerse a participar en todas las convivencias programadas durante el curso, permaneciendo en régimen de internado durante el tiempo de la convivencia. La falta de seriedad y asistencia será un motivo de exclusión.

Llevar adelante los compromisos que se marquen en la convivencia mensual: oración de la mañana y de la tarde, eucaristía, tareas de servicio y estudio...

Mostrar una capacidad normal de convivencia e integración en el grupo, un coeficiente intelectual suficiente y un interés muy especial por la vida de fe. El rendimiento del alumno en su centro de estudios será un signo de interés y seriedad con que se toman la experiencia del Seminario Menor para externos. Si el resultado es habitualmente malo y no se ven signos de recuperación, los alumnos no podrán continuar en la experiencia.

$Y$ hacer un uso cuidadoso de la casa. Ser puntual en la asistencia a los actos que se organicen. Respetar el descanso nocturno... guardar las normas fijadas, respetar a los compañeros y sus bienes, ...

\subsection{Programa educativo:}

"Los contenidos y la metodología de todas las acciones que conforman esta experiencia tienen como objetivo la formación integral cristiana con orientación vocacional:

- el desarrollo de la vida espiritual y sus medios

- la responsabilidad en el estudio

- la integración y colaboración en la parroquia

- la práctica de las virtudes humanas y cristianas

- la participación en la vida familiar

- la sensibilidad a los problemas del mundo

- el conocimiento y el cultivo de la vocación personal"s.

5 Cf. Comisión Episcopal de Seminarios y Universidades, "Habla, Señor". Valor actual del Seminario Menor, 1998, 5 a. 
Al dividir nuestro proyecto en distintas etapas, marcadas por la edad y la situación específica de los chicos, los objetivos formativos quedarían estructurados así: 6

\section{Etapa de sensibilización vocacional ( $6^{\circ}$ de Primaria)}

\section{En lo humano:}

Crear una sana curiosidad e interés por las cosas nuevas.

Impulsar el sentido de pertenencia al grupo de amigos.

Iniciar el conocimiento de las propias capacidades.

\section{En lo cristiano:}

Descubrir a Jesús como amigo.

Tomar conciencia de la presencia de Jesús como vínculo de unión en el grupo de amigos.

Descubrir que el ser amigo de Jesús supone un estilo de vida.

\section{En lo vocacional:}

Descubrir a Jesús como "el que dialoga y llama".

Crear interés por la labor social del grupo de amigos de Jesús.

Descubrir como atractivo el estilo de vida del sacerdote.

\section{La preadolescencia $\left(1^{\circ}\right.$ y $2^{\circ}$ de ESO)}

\section{En lo humano:}

Favorecer el desarrollo de la inteligencia y acrecentar las capacidades de amar y decidir.

Fomentar la apertura a los demás y la capacidad de sociabilidad.

Valorar el propio cuerpo y su significado.

\section{En lo cristiano:}

Aceptar a Jesús como amigo y modelo de identificación, partiendo de la espiritualidad que nace del bautismo.

${ }^{6}$ La distribución de objetivos que aparece a continuación está tomada del Plan de Formación del Seminario de Málaga. 
Experimentar que ser cristiano es pertenecer a una comunidad: la Iglesia. Vivir el ser cristiano como el ser apóstol de Jesús.

En lo vocacional:

Entender la vocación como llamada de Jesús a estar con Él.

Experimentar la vocación como servicio y donación a la comunidad.

Conocimiento de lo específico de la vocación sacerdotal, así como de sus exigencias.

\section{Primera Adolescencia $\left(3^{\circ}\right.$ y $4^{\circ}$ de ESO)}

\section{En lo humano:}

Conocer más la propia personalidad.

Favorecer el proceso de maduración mental, de la afectividad y de la sensibilidad estética.

Potenciar la sana convivencia, el diálogo y la amistad.

\section{En lo cristiano:}

Profundizar en la figura de Jesús, Hijo de Dios, como modelo de hombre.

Ahondar en el amor a la Iglesia de Mérida-Badajoz como realización concreta de la Iglesia universal.

Iniciarse en ser cristiano como apóstol en el propio ambiente.

\section{En lo vocacional:}

Entender la vocación como llamada de Jesús para estar con Él también en el anuncio del Evangelio.

Presentar la vocación como modo de realización personal y como servicio a los demás, especialmente a los más pobres.

Profundizar en la necesidad e importancia de vocaciones en la Iglesia y para el mundo.

\section{Segunda Adolescencia $\left(1^{\circ}\right.$ y $2^{\circ}$ de Bachillerato)}

\section{En lo humano:}

Asumir la tarea en la formación intelectual como un auténtico compromiso. 
Cultivar la afectividad como un valor humano.

Promocionar actividades que den sentido y dinamicen las capacidades de la propia persona.

\section{En lo cristiano:}

Comprender que ser cristiano es ser discípulo de Jesucristo, que se ofreció a sí mismo por los demás.

Comprender que ser cristiano es también dar la vida por y en la Iglesia.

Comprender que ser cristiano es ser testigo de Cristo en el mundo y "militante" en la Iglesia.

\section{En lo vocacional:}

Descubrir la vocación sacerdotal como un estilo de vida apostólico.

Madurar en grupo y personalmente la opción vocacional hacia el ministerio presbiteral.

Prepararse y formarse de acuerdo con la opción por el sacerdocio ministerial.

Todo ello se irá desarrollando conjuntamente a lo largo de los fines de semana en las diversas actividades que se irán desarrollando: charlas, dinámicas, celebraciones, diálogo personal, trabajo en grupo... Así como en las pequeñas acciones y compromisos que realizarán en sus propios lugares de residencia, convivencia y estudio.

\section{Experiencia de profundización vocacional}

\section{Proyecto}

\section{"Campo de Trabajo Buen Samaritano"}

\section{Justificación:}

En esta etapa de profundización proponemos esta actividad, como medio para descubrir la vocación partiendo de experiencias concretas con el mundo de los pobres y alejados. 


\section{Objetivo:}

Poner a los jóvenes ante la realidad que viven los más pobres, donde Dios se hace presente y nos habla. Que escuchen su llamada, den pasos en el descubrimiento de su vocación y lo sigan.

\section{Objetivos específicos}

Conocer la realidad de los más pobres de nuestra Archidiócesis de Mérida-Badajoz.

Que los jóvenes: escuchen, profundicen o descubran su vocación y sigan a Jesucristo que les habla a través de los pobres.

Conocer y valorar el ser y misión de Cáritas.

Realizar una actividad transformadora que repercuta en una mejor de la calidad de vida de los pobres.

Profundizar en nuestra vida cristiana

Celebrar la fe y la vida.

Invitar a que los participantes conozcan y participen en las actividades y vida de sus Cáritas parroquiales.

Que el joven viva la experiencia de la coordinación de distintas áreas de la pastoral eclesial (Pastoral Juvenil-Seminario-Cáritas).

\section{Destinatarios}

Chicos y chicas de 18 a 30 años que sean capaces de plantearse su vida desde la solidaridad con los más necesitados.

\section{Desarrollo.}

Objetivo específico 1: Conocer la realidad de los más pobres de nuestra Archidiócesis de Mérida-Badajoz.

\section{Medios:}

Pedir a Cáritas Diocesana (Juventud-niños) que nos presenten proyectos en los que los jóvenes puedan entrar en contacto con el mundo de la pobreza. 
Que sean proyectos en los que ya se esté y se pueda participar. Si no fuese posible, realizar una actividad que se inicie con este campo de trabajo.

Objetivo específico 2: Que los jóvenes: escuchen, profundicen o descubran su vocación y sigan a Jesucristo que les habla a través de los pobres.

\section{Medios:}

Las celebraciones, oraciones, momentos de formación, etc. se harán en clave vocacional, es decir, que la pregunta "¿qué te está pidiendo Jesucristo?" sea un elemento esencial de este campo de trabajo.

En la presentación del campo de trabajo se hará desde este enfoque (en las actividades que van a realizar, Dios le va a estar hablando).

Objetivo específico 3: Conocer y valorar el ser y misión de Cáritas.

\section{Medios:}

Presentar la actividad a realizar como un proyecto de Cáritas o semejante.

Trabajar tanto en la preparación como en la realización con gente de Cáritas (en lo posible).

Objetivo específico 4: Realizar una actividad transformadora que repercuta en una mejora de la calidad de vida de los pobres.

\section{Medios:}

Que los proyectos en los se colabore sean de carácter promocional y no asistencial (aunque eso se puede replantear según las edades a las que se la ofrezcamos).

Que suponga realizar una actividad (clases de apoyo a niños, acompañar a jóvenes, atender a ancianos, etc.). 
Objetivo específico 5: Profundizar en nuestra vida cristiana.

\section{Medios:}

Tendremos momentos de reflexión y profundización sobre diversos elementos de nuestra fe.

Trabajaremos y reflexionaremos sobre actitudes a desarrollar para tener una acción evangelizadora (para las actividades a realizar en el campo de trabajo como para la vida).

Objetivo específico 6: Celebrar la fe y la vida.

\section{Medios:}

Dedicaremos momentos para la celebración de la eucaristía.

Dedicaremos momentos para la celebración de la penitencia.

Todos los días tendremos tiempo para una oración personal y comunitaria; oración personal, serena, profunda y vital; complementada con momentos para el compartir las vivencias, pedir lo necesario los unos por los otros y agradecer al Señor.

Objetivo específicos 7: Invitar a que los participantes conozcan y participen en las actividades y vida de sus Cáritas parroquiales.

\section{Medios:}

Al final de cada encuentro se trabajará un plan de trabajo con los participantes en el que se concrete cómo poner en práctica en su vida real lo aprendido y vivido.

En este encuentro final se les invitará a conocer qué hace en su parroquia el equipo de cáritas.

Objetivo específico 8: Que el joven viva la experiencia de la coordinación de distintas áreas de la pastoral eclesial (Pastoral Juvenil-Seminario-Cáritas). 


\section{Medios:}

La programación, actividades y calendarios se buscará consensuar entre las delegaciones, secretariados y Seminario, que lo organizan.

Se buscará implicar a cuantas más personas puedan de las distintas realidades que la organizan.

\section{Exigencias:}

Chicos y chicas de entre 18 y 30 años sensibles a la propuesta vocacional en toda su amplitud y dispuestos a descubrirla desde el mundo de los pobres.

Participar de las actividades organizadas durante el año.

\section{Qué les ofrece:}

- Campos de Trabajo

Todo estaría dentro de un mismo programa, que sería desarrollado en dos campos de trabajo anuales.

Estos campos de trabajo se distribuirán de la siguiente manera:

- Del 26 al 30 de diciembre. Desde el acontecimiento de la natividad.

- Desde el sábado anterior al domingo de Ramos hasta el martes santo. Entorno al acontecimiento de la pasión-muerte-resurrección de Jesucristo.

\section{Responsable:}

Responsable será el director de PDAV que coordinará la experiencia y la insertará, desde la etapa de profundización, en el Plan de Diocesano Animación Vocacional.

\section{Colaboradores:}

Caritas. 
Experiencia de profundización vocacional

"POZO DE JACOB"

\section{Justificación:}

Con este proyecto desde el plan diocesano de animación vocacional se intenta dar una respuesta de acompañamiento en profundidad a las chicas que puedan ir surgiendo del Proyecto Samuel o Damasco.

\section{Objetivo:}

Proporcionar formación y acompañamiento, tanto personal como de grupo, a niñas, adolescentes y jóvenes de la comunidad cristiana, para ayudarles a madurar y clarificar su posible vocación a una vida de especial consagración.

\section{Destinatarios:}

Niñas, adolescentes y jóvenes hasta 17 años, que cursan sus correspondientes estudios académicos, viven con sus familias y presentan una inquietud vocacional inicial a la vida consagrada.

\section{Responsable y colaboradores:}

Responsable de la experiencia será la CONFER acompañada por el Delegado Diocesano de Vocaciones.
Acciones:
Encuentros periódicos
Retiros y celebraciones
Cursillo y actividad de verano
Acompañamiento personal.
Lugar de la experiencia:
A determinar por el Arzobispo. 


\section{Experiencia de sensibilización vocacional}

\section{"VENID Y LO VERÉIS"}

\section{Justificación:}

Este proyecto surge desde la apertura que nuestro seminario ha de tener al resto de la Archidiócesis y a todo el trabajo que se realiza con niños, adolescentes y jóvenes.

Sensibilizar a todos los miembros de nuestra iglesia local con el seminario desde la cercanía.

\section{Objetivo:}

Presentar y conocer el Seminario como el corazón de la Archidiócesis y descubrir que desde él parten otras serie de experiencias vocacionales donde chicos y chicas pueden ser protagonistas.

\section{Destinatarios:}

Alumnos de los centros educativos de nuestra Archidiócesis que estén en la asignatura de Religión y grupos de catequesis de las distintas parroquias.

\section{Responsable y colaboradores:}

Responsable de la experiencia será el Rector del Seminario metropolitano.

Contará con la colaboración del resto de los formadores y con la ayuda de los seminaristas del mayor.

\section{Acciones:}

Visita programada al Seminario Metropolitano.

Actividades de conocimiento de su historia y su realidad

Dinámicas vocacionales.

Celebración comunitaria de la Palabra 


\section{Lugar de la experiencia:}

Seminario Metropolitano

\section{Experiencia}

\section{"NICODEMO"}

\section{Justificación:}

Este proyecto surge desde la necesidad que manifiesta el presbiterio diocesano de ilusionarse desde la propia vocación para contagiar a los demás en este estilo de vida. Trabajar todos juntos desde principios iguales en lo que en nuestra diócesis es la llamada Vocacional.

\section{Objetivo:}

- Situar a los sacerdotes ante su realidad de personas llamadas por Dios a vivir su vocación en el ministerio ordenado. A re-ilusionarse con la vocación recibida para ilusionar a los demás y poder dar pasos.

\section{Destinatarios:}

Sacerdotes de nuestra Diócesis, dispuestos a pararse y redescubrir su ser ministerial como personas llamadas por Dios.

\section{Responsable y colaboradores:}

Responsable de la experiencia será el delegado del Clero junto con el Responsable del PDAV.

Contarán con la colaboración del resto de los formadores y con la ayuda de sacerdotes implicados en esta experiencia. 
Acciones:

Material para oración personal.

Material para trabajo en grupo.

Escritos ministeriales

Jornada de profundización. 